The Bulletin of Symbolic Logid

Volume 8, Number 4, Dec. 2002

\title{
COMPUTABILITY-THEORETIC COMPLEXITY OF COUNTABLE STRUCTURES
}

\author{
VALENTINA S. HARIZANOV*
}

\section{CONTENTS}

1. Introduction

2. Elementary and atomic diagrams of prime, saturated, and other countable models

3. Complexity of diagrams of models of arithmetic 464

4. Turing degrees of isomorphism types 467

5. n-diagrams of countable structures 470

§1. Introduction. Computable model theory, also called effective or recursive model theory, studies algorithmic properties of mathematical structures, their relations, and isomorphisms. These properties can be described syntactically or semantically. One of the major tasks of computable model theory is to obtain, whenever possible, computability-theoretic versions of various classical model-theoretic notions and results. For example, in the 1950's, Fröhlich and Shepherdson realized that the concept of a computable function can make van der Waerden's intuitive notion of an explicit field precise. This led to the notion of a computable structure. In 1960, Rabin proved that every computable field has a computable algebraic closure. However, not every classical result "effectivizes". Unlike Vaught's theorem that no complete theory has exactly two nonisomorphic countable models, Millar's and Kudaibergenov's result establishes that there is a complete decidable theory that has exactly two nonisomorphic countable models with computable elementary diagrams. In the 1970's, Metakides and Nerode [58], [59] and Remmel [71], [72], [73] used more advanced methods of computability theory to investigate algorithmic properties of fields, vector spaces, and other mathematical structures. At the same time and independently, computable

Received February 18, 2002; accepted June 18, 2002.

${ }^{*}$ I am very thankful to R. Soare and the referee for their contributions and valuable suggestions. 
model theory was developed by the Russian school of constructive mathematics (see [19]).

We consider only countable structures for computable languages. A computable language is a countable language with algorithmically presented set of symbols and their arities. The universe $A$ of an infinite countable structure $\mathcal{A}$ can be identified with $\omega$. As usual, if $L$ is the language of $\mathcal{A}$, then $L_{A}$ is the language $L$ expanded by adding a constant symbol for every $a \in A$, and $\mathcal{A}_{A}=(\mathcal{A}, a)_{a \in A}$ is the corresponding expansion of $\mathcal{A}$ to $L_{A}$. We say that a first-order formula is $\Sigma_{0}\left(\right.$ or $\left.\Pi_{0}\right)$ if it is quantifier-free. For $n>0$, a formula in prenex normal form is $\Sigma_{n}\left(\Pi_{n}\right.$, respectively) if it has $n$ blocks of like quantifiers, beginning with $\exists\left(\forall\right.$, respectively). A $B_{n}$ formula is a Boolean combination of $\Sigma_{n}\left(\right.$ or $\left.\Pi_{n}\right)$ formulae. A relation is $\Sigma_{0}\left(\right.$ or $\left.\Pi_{0}\right)$ if it is computable. As for formulae, we define $\Sigma_{n}$ and $\Pi_{n}$ relations. A relation is arithmetical if it is $\Sigma_{n}$ for some $n$. A relation is $\Delta_{n}$ if it is both $\Sigma_{n}$ and $\Pi_{n}$. We use $\leq_{T}$ for Turing reducibility and $\equiv_{T}$ for Turing equivalence of sets. By $\operatorname{deg}(X)$ we denote the Turing degree of $X$. For $X, Y \subseteq \omega$, the join of $X$ and $Y$ is $X \oplus Y=\{2 m: m \in X\} \cup\{2 m+1: m \in Y\}$. By $X^{(n)}$ we denote the $n$th jump of $X$, and by $X^{(\omega)}$ its $\omega$-jump. The Turing degree $\operatorname{deg}\left(X^{(\alpha)}\right)$ is also denoted by $\mathbf{x}^{(\alpha)}$. The degree $\mathbf{0}^{(\omega)}$ is a natural upper bound for the sequence $\left(\mathbf{0}^{(n)}\right)_{n \in \omega}$, although no ascending sequence of Turing degrees has a least upper bound. Post proved that a relation $R$ is $\Delta_{n+1}$ iff $R \leq_{T} \emptyset^{(n)}$. Post also proved that a relation is $\Sigma_{n+1}$ iff it is computably enumerable (c.e.) in a $\Pi_{n}$ relation.

A theory in $L$ is a consistent set of sentences in $L$. A complete type (or, briefly, a type) is a maximal consistent set of formulae in a certain fixed number of variables. We will often identify a formula $\theta$ with its Gödel number $\lceil\theta\rceil$. We say that a set $\Gamma$ of formulae belongs to a certain computabilitytheoretic complexity class $\mathcal{C}$ if $\{\lceil\theta\rceil: \theta \in \Gamma\} \in \mathcal{C}$. Hence, a theory is computable, or decidable, if the set of its theorems is computable. Clearly, a computably axiomatizable theory, namely a theory whose set of theorems is c.e., which is also a complete theory, is decidable. Kleene, and also Hasenjaeger, showed that if $T$ is a computably axiomatizable theory, then $T$ has a model whose domain is a set of natural numbers, such that every relation and function of the model is $\Delta_{2}$. On the other hand, Kreisel, Mostowski, and Putnam (independently) showed that there is a computably axiomatizable theory that does not have a model in which every relation and function is c.e. or co-c.e.

The atomic (open) diagram of a structure $\mathcal{A}$ is the set of all quantifier-free sentences of $L_{A}$ true in $\mathcal{A}_{A}$. A structure is computable if its atomic diagram is computable. The structure $\mathcal{N}=(\omega,+, \cdot, S, 0)$ is computable. A standard model of arithmetic is a structure isomorphic to $\mathcal{N}$. Tennenbaum showed that there is no computable nonstandard model of Peano Arithmetic, PA. 
Moreover, in a nonstandard model of $P A$, neither addition, nor multiplication is computable (see [40]). The Turing degree of $\mathcal{A}, \operatorname{deg}(\mathcal{A})$, is the Turing degree of the atomic diagram of $\mathcal{A}$. Hence, $\mathcal{A}$ is computable iff $\operatorname{deg}(\mathcal{A})=\mathbf{0}$. Lerman and Schmerl [54] proved that every $\Sigma_{2}$ theory of linear order has a computable model. On the other hand, they showed that there is a complete $\Delta_{3}$ theory of linear order without a computable model. Shoenfield improved Hasenjager's and Kleene's result by establishing that a computably axiomatizable theory has a model whose Turing degree is $<\mathbf{0}^{\prime}$. For example, Shoenfield's result implies that there is a nonstandard model of $P A$ whose Turing degree is $<\mathbf{0}^{\prime}$.

The elementary (complete) diagram of $\mathcal{A}, D^{c}(\mathcal{A})$, is the set of all sentences of $L_{A}$ that are true in $\mathcal{A}_{A}$. A structure $\mathcal{A}$ is decidable if its complete diagram $D^{c}(\mathcal{A})$ is computable. In other words, a structure $\mathcal{A}$ is decidable if there is an algorithm that determines for every formula $\theta\left(x_{0}, \ldots, x_{n-1}\right)$ and every sequence $\left(a_{0}, \ldots, a_{n-1}\right) \in A^{n}$, whether $\mathcal{A}_{A} \quad \theta\left(a_{0}, \ldots, a_{n-1}\right)$. For example, the linear order of rationals is a decidable model of the theory of dense linear order without endpoints. Let $T$ be a complete theory in $L$ and $\mathcal{A}$ a decidable model of $T$. Then for every sentence $\sigma$ in $L,[T \vdash \sigma] \Leftrightarrow[\mathcal{A} \quad \sigma]$, so $T$ is a decidable theory. Moreover, every type of $T$ realized in $\mathcal{A}$ is computable. The set of all types of $T$ realized in $\mathcal{A}$ is uniformly computable. Henkin's construction of models is effective and yields the following result.

Effective COMPLeteness Theorem. A decidable theory has a decidable model.

Clearly, every decidable structure is computable. The converse is not true. For example, the structure $\mathcal{N}$ is computable, but not decidable. However, if a theory admits effective quantifier elimination, then every computable model of the theory is decidable. Thus, every computable model of the theory of algebraically closed fields of characteristic 0 is decidable. Similarly, every computable model of the theory of dense linear order without endpoints is decidable.

By $f: \mathcal{A} \cong \mathcal{B}$ we denote that $f$ is an isomorphism from $\mathcal{A}$ onto $\mathcal{B}$. We call any structure isomorphic to $\mathcal{A}$ an isomorphic copy (or, briefly, a copy) of $\mathcal{A}$. Hence, an $\aleph_{0}$-categorical theory $T$ with only infinite models is decidable iff every countable model of $T$ has a decidable isomorphic copy. Harrington [33] and Khisamiev [41] established that every countable model of a decidable $\aleph_{1}$-categorical theory has a decidable copy. We define the $n$-diagram of $\mathcal{A}, D_{n}(\mathcal{A})$, to be the set of all $\Sigma_{n}$ sentences of $L_{A}$ that are true in $\mathcal{A}_{A}$. In particular, $D_{0}(\mathcal{A})$ is the open diagram of $\mathcal{A}$. There are familiar structures for which Turing degrees of the $n$-diagrams are strictly increasing. For example, the $n$-diagram of $\mathcal{N}$ has Turing degree $\mathbf{0}^{(n)}$. For $n \geq 1$, a structure is $n$-decidable if its $n$-diagram is computable. Moses [67] showed that for every $n \geq 1$, there is a linear order that is $n$-decidable, but has no $(n+1)$-decidable copy. Chisholm and Moses [12] have shown 
that there is a linear order that is $n$-decidable for every $n \in \omega$, but has no decidable copy. Goncharov [22] established similar results for Boolean algebras.

For sets $X$ and $Y$, we say that $Y$ is c.e. in and above (c.e.a. in) $X$ if $Y$ is c.e. relative to $X$, and $X \leq_{T} Y$. For any structure $\mathcal{A}, D_{n+1}(\mathcal{A})$ is c.e.a. in $D_{n}(\mathcal{A})$, uniformly in $n$. (This would not be true if we had defined $D_{n}(\mathcal{A})$ as $D^{c}(\mathcal{A}) \cap B_{n}$.) Clearly, $D_{n}(\mathcal{A}) \equiv_{T} D^{c}(\mathcal{A}) \cap B_{n}$. An $\omega$-table is a sequence of sets $\left(C_{n}\right)_{n \in \omega}$, where $C_{n+1}$ is c.e.a. in $C_{n}$, uniformly in $n$. The $\omega$-table is said to be over $X$ if $C_{0}=X$. Similarly, for $k \in \omega-\{0\}$, a $k$-table is a sequence $\left(C_{n}\right)_{n<k}$ such that for $n+1<k, C_{n+1}$ is c.e.a. in $C_{n}$. (In [5], Ash and Knight introduced the notion of an $\alpha$-table for any computable ordinal $\alpha \geq 1$.) For every structure $\mathcal{A},\left(D_{n}(\mathcal{A})\right)_{n \in \omega}$ is an $\omega$-table. Harizanov, Knight and Morozov [32] investigated possible sequences of Turing degrees of $n$-diagrams for $\mathcal{B} \simeq \mathcal{A},\left(\operatorname{deg}\left(D_{n}(\mathcal{B})\right)\right)_{n \in \omega}$.

It is easy to see that the theory of a structure $\mathcal{A}$ is computable in $D^{c}(\mathcal{A})$, and that $D^{c}(\mathcal{A})$ is computable in $\left(D_{0}(\mathcal{A})\right)^{(\omega)}$. The atomic diagram of a model of a theory may be of much lower Turing degree than the theory itself. For example, true arithmetic, $T A$, is the theory of $\mathcal{N}$, and its Turing degree is $\mathbf{0}^{(\omega)}$. For any theory $S$, Henkin's construction produces a model $\mathcal{H}$ with $D^{c}(\mathcal{H}) \leq_{T} S^{\prime}$. A set $X \leq_{T} \emptyset^{\prime}$ and its Turing degree $\mathbf{x}$ are called low if $\boldsymbol{x}^{\prime} \leq \mathbf{0}^{\prime}$, and low if $\boldsymbol{x}^{(\boldsymbol{n})} \leq \mathbf{0}^{(\boldsymbol{n})}$. The Low Basis Theorem of Jockusch and Soare [38], establishes that every infinite binary tree $\Lambda$ has an infinite path $H$ with $H^{\prime} \leq_{T} \Lambda^{\prime}$. In particular, every infinite computable binary tree has a low path. The Low Basis Theorem can be used to obtain for a theory $S$, a model $\mathcal{H}$ with $\left(D^{c}(\mathcal{H})\right)^{\prime} \leq_{T} S^{\prime}$, thus strengthening Shoenfield's result. As a general source for model theory we use [10] and [9], for computability theory [78], and for computable model theory [6], [20], [31] and [63].

§. Elementary and atomic diagrams of prime, saturated, and other countable models. Every computable type of a complete decidable theory is realized in some decidable model of that theory. The set of all computable types of a complete decidable theory is a $\Pi_{2}$ set. Every principal type of such a theory is computable, and the set of all its principal types is $\Pi_{1}$. However, if a complete decidable theory $T$ has a decidable prime model, then the set of all principal types of $T$ is uniformly computable. The following theorem establishes the converse. Its proof combines the Henkin method of constructing models with the finite injury priority method.

Theorem 2.1 (Goncharov and Nurtazin [29], Harrington [33]). Let T be a complete decidable theory. Then the following are equivalent:

(a) The theory $T$ has a decidable prime model.

(b) The theory $T$ has a prime model and the set of all principal types of $T$ is uniformly computable. 
(c) There is a computable function which maps the Gödel code of every formula consistent with $T$ to a Gödel index of a computable principal type of $T$ containing the formula.

Peretyat'kin [69] showed that there is a complete atomic finitely axiomatizable theory without a computable prime model. Hirschfeldt [35] has proven that there is a complete theory of linear order with a prime model and a computable model, but without a computable prime model. Previously, Khisamiev established a similar result for Abelian groups.

On the other hand, Millar [62] showed that a complete atomic decidable theory has a prime model $\mathcal{A}$ such that $D^{c}(\mathcal{A}) \leq_{T} \emptyset^{\prime}$. Csima has recently strengthened this result by establishing the Prime Model Low Basis Theorem. This theorem does not follow from the Low Basis Theorem of Jockusch and Soare.

THEOREM 2.2 (Csima [14], [13]). Let $T$ be a complete atomic decidable theory.

(a) The theory $T$ has a prime model $\mathcal{A}$ such that $D^{c}(\mathcal{A})$ is low.

(b) The theory $T$ has low prime models $\mathcal{A}$ and $\mathcal{B}$ such that their Turing degrees form a minimal pair.

Csima also established the Prime Model Avoiding Cones Theorem, i.e., she proved that no nontrivial information can be coded into all prime models of $T$.

THEOREM 2.3 (Csima [14], [13]). Let T be a complete atomic decidable theory. For every noncomputable set $X$, there is a prime model $\mathcal{A}$ of $T$ with $X \quad{ }_{T} D^{c}(\mathcal{A})$, and indeed $\mathcal{A}$ can even be chosen to be low.

In [15], a set $X$ is called prime bounding if every complete atomic decidable theory $T$ has a prime model $\mathcal{A}$ such that $D^{c}(\mathcal{A}) \leq_{T} X$. For example, it follows from Millar's result that $\emptyset^{\prime}$ is prime bounding.

Theorem 2.4 (Csima, Hirschfeldt, Knight and Soare [15]). Let $X \leq_{T} \emptyset^{\prime}$. Then $X$ is prime bounding if and only if $X$ is not low 2 .

To prove that a $l_{\text {low }}$ set $X$ is not prime bounding, one uses a $\emptyset^{\prime}$-computable listing of the array of sets $\left\{Y: Y \leq_{T} X\right\}$ to find a complete atomic decidable theory $T$, which diagonalizes against all potential prime models of $T$ whose elementary diagrams are computable in $X$. To prove that any set $X$ that is not low 2 is indeed prime bounding, one fixes a function $f \leq_{T} X$ that dominates every total $\emptyset^{\prime}$-computable function. Given a complete atomic decidable theory $T$, one uses $f$ to build a prime model of $T$.

Millar [61] proved that a complete decidable theory $T$ without a decidable prime model has infinitely many pairwise nonisomorphic decidable models, such that the set of all types realized in any two of these models simultaneously is exactly the set of all principal types of $T$. This result can be obtained using the following Effective Omitting Types Theorem. 
THEOREM 2.5 (Millar [61]). Let $T$ be a complete decidable theory, and let $\Phi$ be a $\Sigma_{2}$ set of computable types of $T$. Then $T$ has a decidable model that omits every nonprincipal type in $\Phi$.

Millar showed that the previous theorem does not hold if $\Phi$ contains computable partial types of $T$, even if $\Phi$ is a computable set. Csima has shown that for such computable partial types, Millar's degree $\mathbf{0}$ is the only $\Delta_{2}$ Turing degree ruled out as the degree of the elementary diagram of a model omitting all types.

THEOREM 2.6 (Csima [13]). Let $T$ be a complete decidable theory, and $\Phi$ a computable set of computable partial types of $T$. Then for every noncomputable $\Delta_{2}$ set $X$, there is a model $\mathcal{A}$ of $T$ such that $D^{c}(\mathcal{A}) \equiv_{T} X$ and $\mathcal{A}$ omits every nonprincipal type in $\Phi$.

Millar [62] was first to show that there is a complete atomic decidable theory with all types computable, but no computable prime model. Csima established the following basis theorem for prime models of such theories.

THEOREM 2.7 (Csima [13]). Let $T$ be a complete atomic decidable theory with all types computable. For every noncomputable $\Delta_{2}$ set $X$, there is a prime model $\mathcal{A}$ of $T$ such that $D^{c}(\mathcal{A}) \equiv_{T} X$.

If all types of a complete decidable theory are computable, then $T$ has a countable saturated model. However, Millar [62] constructed a complete decidable theory $T$ whose all types are computable, such that $T$ does not have a computable saturated model. If $T$ has a decidable saturated model, then the types of $T$ are uniformly computable. The converse is also true.

Theorem 2.8 (Morley [65], Millar [62]). Let $T$ be a complete decidable theory such that the set of all types of $T$ is uniformly computable. Then $T$ has a decidable saturated model.

Therefore, a complete theory with a decidable saturated model also has a decidable prime model. Millar [60] showed that there is a homogeneous model $\mathcal{A}$ whose set of all types is uniformly computable, but $\mathcal{A}$ does not have a decidable copy. On the other hand, Millar [62] established that for such a model $\mathcal{A}$, we must have $D^{c}(\mathcal{A}) \leq_{T} \emptyset^{\prime}$.

Goncharov and Peretyat'kin independently generalized Theorems 2.1 and 2.8 by giving a criterion for the existence of a decidable copy of a countable homogeneous structure $\mathcal{A}$. The criterion involves an effective extension of every type realized in $\mathcal{A}$ by adding a new consistent formula with an additional variable.

TheORem 2.9 (Goncharov [24], Peretyat'kin [70]). Let $\mathcal{A}$ be a homogeneous structure. Then $\mathcal{A}$ has a decidable copy if and only if there is a computable enumeration $\Gamma_{0}\left(\bar{u}_{0}\right), \Gamma_{1}\left(\bar{u}_{1}\right), \Gamma_{2}\left(\bar{u}_{2}\right), \ldots$ of all types realized in $\mathcal{A}$, and a computable binary function $f$ such that if $(\exists x) \theta\left(\bar{u}_{i}, x\right) \in \Gamma_{i}\left(\bar{u}_{i}\right)$, then for 
$j=f(i,\lceil\theta\rceil)$, we have that $\bar{u}_{j}=\left(\bar{u}_{i}, x\right)$ and

$$
\Gamma_{i}\left(\bar{u}_{i}\right) \cup\left\{\theta\left(\bar{u}_{i}, x\right)\right\} \subseteq \Gamma_{j}\left(\bar{u}_{j}\right) .
$$

Macintyre and Marker [55] have a relativized version of the previous theorem (also, see [26]).

Lerman and Schmerl investigated $\aleph_{0}$-categorical theories with computable models.

THEOREM 2.10 (Lerman and Schmerl [54]). Let $T$ be an arithmetical $\aleph_{0^{-}}$ categorical theory such that for every $n \in \omega$, the fragment $T \cap \Sigma_{n+2}$ is $\Sigma_{n+1}$. Then $T$ has a computable model.

Lerman and Schmerl [54] also showed that for every $n \in \omega$ and a Turing degree $\mathbf{d} \mathbf{0}^{(n)}$, there is an $\aleph_{0}$-categorical theory $T$ of degree $\mathbf{d}$ without a computable model, such that $T \cap \Sigma_{n+1}$ is computable.

Goncharov [23] and Kudaibergenov [51] showed that for any $n \geq 1$, there is an $\aleph_{1}$-categorical but not totally categorical theory $T$ such that only the first $n$ models in the Baldwin-Lachlan elementary chain of countable models of $T$ have computable copies.

Theorem 2.11 (Khoussainov, Nies and Shore [42]). (a) There is an $\aleph_{1}$ categorical but not totally categorical theory such that all its models in the Baldwin-Lachlan elementary chain, except the prime one, have computable copies.

(b) There is an $\aleph_{1}$-categorical but not totally categorical theory such that all its models in the Baldwin-Lachlan elementary chain, except the saturated one, have computable copies.

The $\aleph_{1}$-categorical theories in the previous examples are all strongly minimal. That is, every definable (with parameters) subset of the domain of any model of such a theory is finite or cofinite. A structure is strongly minimal if its theory is strongly minimal. Nies [68] also showed that there is a strongly minimal theory $T$ such that the only model in the Baldwin-Lachlan elementary chain with a computable copy is the second one. Hence, $T$ does not have a computable prime, nor a computable saturated model. The spectrum of computable models of an $\aleph_{1}$-categorical but not totally categorical theory $T$ is defined to be

$$
\left\{\alpha \leq \omega: \mathcal{A}_{\alpha} \text { has a computable copy }\right\},
$$

where $\left(\mathcal{A}_{\alpha}\right)_{\alpha \leq \omega}$ is the Baldwin-Lachlan elementary chain of countable models of $T$. Hence, some of the previous results can be restated as results about the possible spectra of computable models. Herwig, Lempp and Ziegler [34] were first to find such a spectrum, different from $\omega \cup\{\omega\}$, for a theory in a finite language.

A strongly minimal model $\mathcal{A}$ is trivial if it has trivial pregeometry, that is, for every subset $X \subseteq A$, the algebraic closure of $X$ is the union of the 
algebraic closures of all elements in $X$. Triviality is indeed a property of the theory of a model.

THeorem 2.12 (Goncharov, Harizanov, Laskowski, Lempp and McCoy [27]). Let $T$ be a trivial, strongly minimal theory with at least one computable model. Then every countable model of $T$ has a copy $\mathcal{A}$ such that $D^{c}(\mathcal{A}) \leq_{T} \emptyset^{\prime \prime}$. The spectrum of computable models of $T$ is a $\Sigma_{5}$ subset of $\omega \cup\{\omega\}$.

Similar questions remain open for nontrivial, strongly minimal theories. Goncharov and Khoussainov [28] have shown that for every $n \in \omega$, there is a non-strongly minimal, trivial, $\aleph_{1}$-categorical theory of Turing degree $\mathbf{0}^{(n)}$, all of whose countable models have computable copies. Theorem 2.12 follows from a purely model-theoretic result in [27], that for a trivial, strongly minimal theory $T$ in a language $L$, the elementary diagram of any model $\mathcal{A}$ of $T$ is a model complete $L_{A}$-theory. It can then be shown that $T$ is $\Sigma_{3}$ axiomatizable. On the other hand, Marker [57] previously showed that there is an almost strongly minimal, $\aleph_{0}$-categorical theory, which is not $\Sigma_{n}$ axiomatizable for every $n$.

§3. Complexity of diagrams of models of arithmetic. Jockusch and Soare [38] showed that there is a nonstandard model of $P A$ of low Turing degree. Using his workers method for nested priority constructions, Harrington proved that there is an arithmetical, even $\Delta_{2}$, nonstandard model $\mathcal{A}$ of $P A$ whose theory is not arithmetical. Harrington's construction of $\mathcal{A}$ exploits infinitely many workers. For $n>1$, the $n$th worker produces $D_{n}(\mathcal{A})$. It has an access to the oracle $\emptyset^{(n)}$, and always guesses what the $(n+1)$ st worker has done. Knight further improved Harrington's result.

THEOREM 3.1 (Harrington, Knight [6]). There is a nonstandard model $\mathcal{A}$ of $P A$ such that $T A \leq_{T} T h(\mathcal{A})$ and $D_{0}(\mathcal{A})$ is low.

Solovay and Marker [56] were first to show that for a nonstandard model $\mathcal{A}$ of $P A$, the set of Turing degrees of all isomorphic copies of $\mathcal{A}$ is closed upwards. In particular, the set of Turing degrees of all nonstandard models of $T A$ is closed upwards. Knight showed that if $\mathcal{A}$ is a nonstandard model of $P A$, then the set of Turing degrees of its isomorphic copies has no minimal element.

THEOREM 3.2 (Knight [49]). If $\mathcal{A}$ is a nonstandard model of $P A$, then there exists $\mathcal{B} \cong \mathcal{A}$ such that $D_{0}(\mathcal{B})<_{T} D_{0}(\mathcal{A})$.

It is not known whether the previous result can be strengthened to obtain both $D_{0}(\mathcal{B})<_{T} D_{0}(\mathcal{A})$ and $D_{1}(\mathcal{A}) \leq_{T} D_{1}(\mathcal{B})$.

Every model of $P A$ has an isomorphic copy that has no increase in Turing degree-theoretic complexity between its $n$-diagram and $(n+1)$-diagram, for any $n$. However, the following theorem shows that there is no nonstandard model of $P A$ that intrinsically shows no increase in such complexity. 
TheOREM 3.3 (Knight [45]). Let $\mathcal{A}$ be a nonstandard model of PA. Then there is $\mathcal{B} \cong \mathcal{A}$ such that

$$
D_{0}(\mathcal{B})<_{T} D_{1}(\mathcal{B})<_{T} D_{2}(\mathcal{B})<_{T} \ldots
$$

The proof involves forcing, a weak saturation property of nonstandard models of $P A$, and a new consistency result.

Feferman [21] proved that every arithmetical set is computable in the atomic diagram of any nonstandard model of $T A$. Knight showed that there is a nonstandard model of $T A$ of the Turing degree $<\mathbf{0}^{(\omega)}$ (see [44]). Marker [56] proved that for a Turing degree $\mathbf{d}$ with $\mathbf{d}>\mathbf{0}^{(n)}$ for every $n \geq 0$, there is a nonstandard model $\mathcal{A}$ of $T A$ such that $\operatorname{deg}(\mathcal{A}) \leq \mathbf{d}^{\prime}$. Knight, Lachlan and Soare [44] improved Marker's result by obtaining $(\operatorname{deg}(\mathcal{A}))^{\prime} \leq \mathbf{d}^{\prime}$. As a corollary, they deduced that there is a nonstandard model of $T A$ of Turing degree $\mathbf{d}$ such that $\mathbf{d}^{\prime \prime}=\mathbf{0}^{(\omega)}$. Knight, Lachlan and Soare also refuted the conjecture that the converse of Feferman's theorem holds.

TheOREM 3.4 (Knight, Lachlan and Soare [44]). There is a Turing degree d such that $\mathbf{d}>\mathbf{0}^{(n)}$ for every $n \in \omega$, but $\mathbf{d}$ is not the Turing degree of a nonstandard model of TA.

We now introduce Scott sets, named after Dana Scott [75], which are naturally associated with the completions of $P A$. Let $\left(\tau_{n}\right)_{n \in \omega}$ be a fixed computable 1-1 enumeration of all sequences (nodes) in $2^{<\omega}$. (We identify a sequence $\tau_{n}$ with its index $n$.) A tree $\mathcal{T}_{A}$, for a set $A \subseteq \omega$, is a set of nodes $\left\{\tau_{n}: n \in A\right\}$ that is closed under initial segments in $2^{<\omega}$. A path $P$ on a tree $\mathcal{T}$ is a maximal linearly ordered set of nodes of $\mathcal{T}$ closed under initial segments in $2^{<\omega}$. Let $[\mathcal{T}]$ denote the set of all infinite paths on $\mathcal{T}$.

DEFINITION 1. A Scott set is a nonempty family $\mathcal{S} \subseteq \mathcal{P}(\omega)$ such that for all $A, B \subseteq \omega$ :

(i) $(A \in \mathcal{S} \wedge B \in \mathcal{S}) \Longrightarrow A \oplus B \in \mathcal{S}$,

(ii) $\left(A \in \mathcal{S} \wedge B \leq_{T} A\right) \Longrightarrow B \in \mathcal{S}$,

(iii) $\left(A \in \mathcal{S} \wedge \mathcal{T}_{A}\right.$ an infinite tree $) \Longrightarrow(\exists P \in \mathcal{S})\left(P \in\left[\mathcal{T}_{A}\right]\right)$.

Another way to look at condition (iii) of the previous definition is that a consistent set of axioms in $\mathcal{S}$ has a completion in $\mathcal{S}$. For example, the set of all arithmetical sets is a Scott set. Another important example of a Scott set for every nonstandard model $\mathcal{A}$ of $P A$ is the standard system of $\mathcal{A}$ :

$\left\{\left\{n \in \omega: \mathcal{A}_{A} \quad\right.\right.$ " $a$ is divisible by the $n$th prime number" $\left.\} \mid a \in A\right\}$.

We say that a set $X \subseteq \omega$ is representable in a model $\mathcal{A}$ of $P A$ if there is a formula $\psi(x)$ in the language of $P A$, such that

$$
X=\left\{n: \mathcal{A} \quad \psi\left(S^{n}(0)\right)\right\} .
$$

TheOREM 3.5 (Scott [75]). If $\mathcal{A}$ is a model of PA, then $\{X \subseteq \omega: X$ is representable in $\mathcal{A}\}$ is a Scott set. Conversely, for any countable Scott set 
$\mathcal{S}$, there is a nonstandard model $\mathcal{A}$ of $P A$ such that $\mathcal{S}=\{X \subseteq \omega: X$ is representable in $\mathcal{A}\}$.

An enumeration of a countable set $\mathcal{S} \subseteq \mathcal{P}(\omega)$ is a binary relation $v$ such that $\mathcal{S}=\left\{v_{0}, v_{1}, v_{2}, \ldots\right\}$, where for every $i \in \omega, v_{i}={ }_{\text {def }}\{n:(i, n) \in v\}$. The number $i$ is called a $v$-index of the set $v_{i}$.

Definition 2. Let $\mathcal{S}$ be a countable Scott set. An effective enumeration of $\mathcal{S}$ is an enumeration $v$ with associated binary functions $f, g$, and a unary function h such that for every $i, j \in \omega$ :

(i) $v_{i} \oplus v_{j}=v_{f(i, j)}$,

(ii) $\left(v_{i}=A \wedge B=\{e\}^{A}\right) \Longrightarrow B=v_{g(i, e)}$,

(iii) $\mathcal{T}_{v_{i}}$ an infinite tree $\Longrightarrow v_{h(i)} \in\left[\mathcal{T}_{v_{i}}\right]$.

This effective enumeration is computable in a set $X \subseteq \omega$ if $v, f, g, h$ are all computable in $X$.

Solovay proved that the Turing degrees of nonstandard models of $T A$ are precisely the Turing degrees of effective enumerations of Scott sets containing the arithmetical sets (see [48]). However, Macintyre and Marker [55], using Theorem 2.9 relativized to an arbitrary oracle $X$, showed that if a countable Scott set $\mathcal{S}$ has an enumeration computable in $X$, then $\mathcal{S}$ has an effective enumeration computable in $X$. Hence, in Solovay's characterization of Turing degrees of nonstandard models of $T A$, "effective enumeration" can be replaced by "enumeration".

Theorem 3.6 (Solovay, Marker). For a Turing degrees $\mathbf{d}$, the following are equivalent:

(i) There is a nonstandard model $\mathcal{A}$ of TA such that $\operatorname{deg}(\mathcal{A})=\mathbf{d}$;

(ii) For some Scott set containing all arithmetical sets, there is an enumeration $v$ such that $\operatorname{deg}(v)=\mathbf{d}$.

We say that a Turing degree $\mathbf{d}$ is a uniform upper bound for the arithmetical sets if there is an enumeration $v$ of the set of all arithmetical sets such that $\operatorname{deg}(v) \leq \mathbf{d}$. Hence, every uniform upper bound for the arithmetical sets is the Turing degree of a nonstandard model of $T A$. On the other hand, Lachlan and Soare [52] proved that there is a model of $T A$ whose Turing degree is not a uniform upper bound for the arithmetical sets. A Turing degree $\mathbf{d}$ is a subuniform upper bound for the arithmetical sets if there is an enumeration $v$ of sets containing all arithmetical sets such that $\operatorname{deg}(v) \leq \mathbf{d}$. If $\mathcal{A}$ is a nonstandard model of $T A$ of Turing degree $\mathbf{d}$, then $\mathbf{d}$ is a subuniform upper bound for the arithmetical sets. However, the converse does not hold. Lachlan and Soare [53] proved that there is a Turing degree that is a subuniform upper bound for the arithmetical sets, but is not the Turing degree of a nonstandard model of $T A$.

Solovay also characterized the Turing degrees of nonstandard models of arbitrary completions of $P A$ (see [48] and [2]). Arana further extended Solovay's result to $n$-diagrams. 
THEOREM 3.7 (Arana [1]). Let $C$ be a completion of $P A$, and $n \in \omega$. Then the following are equivalent for a set $X \subseteq \omega$ :

(i) There is a nonstandard model $\mathcal{A}$ of $C$ such that $D_{n}(\mathcal{A}) \equiv_{T} X$;

(ii) For some $S$ cott set $\mathcal{S}$ with $C \cap \Sigma_{i} \in \mathcal{S}$ for every $i$, there is an enumeration $v \leq_{T} X$, and a sequence of unary functions $\left(f_{n+i}\right)_{i \geq 1}$ such that

- $f_{n+i}$ is $\Delta_{i}$ in $X$, uniformly in $i$,

- $\lim _{s \rightarrow \infty} f_{n+i}(s)$ is a v-index for $C \cap \Sigma_{n+i}$,

- for every $s, f_{n+i}(s)$ is a $v$-index for a subset of $C \cap \Sigma_{n+i}$.

A related open problem is to characterize sequences of Turing degrees of $n$-diagrams for models of a given completion of $P A$. Arana characterized Turing degrees of $n$-diagrams of nonstandard models of $T A$.

Theorem 3.8 (Arana [1]). For every $n \in \omega$, the Turing degrees of $n$ diagrams of nonstandard models of TA are the Turing degrees of enumerations of Scott sets containing the arithmetical sets.

Hence, the Turing degrees of $n$-diagrams of nonstandard models of $T A$ are the same for every $n \in \omega$.

$\S 4$. Turing degrees of isomorphism types. Consider $\mathcal{A}=(\omega,<)$. Then for any Turing degree $\mathbf{d}$, there is a structure $\mathcal{B}=(\omega, \prec)$ isomorphic to $\mathcal{A}$ such that $\operatorname{deg}(\mathcal{B})=\mathbf{d}$. This fact is easily established via coding any set $X$ into $\mathcal{B}$ by arranging that

$$
\begin{array}{ll}
2 n \prec 2 n+1 & \text { if } n \in X, \\
2 n+1 \prec 2 n & \text { if } n \notin X .
\end{array}
$$

The Turing degree spectrum of a countable structure $\mathcal{A}$ is

$$
\operatorname{DgSp}(\mathcal{A})=\{\operatorname{deg}(\mathcal{B}): \mathcal{B} \cong \mathcal{A}\} .
$$

Slaman [76] and Wehner [80] have independently constructed examples of Turing degree spectra of structures consisting of all nonzero Turing degrees. This is not always the case. Downey and Jockusch [16] have shown that any Boolean algebra of low Turing degree has a computable copy, and Thurber [79], and Knight and Stob [50] have further extended this result to Boolean algebras of $l o w_{2}$ and $l o w_{4}$ Turing degrees, respectively. They have conjectured that a similar result holds for Boolean algebras of any low $w_{n}$ Turing degree.

Jockusch and Soare [39] proved that for every nonzero c.e. Turing degree d, there is a linear order of Turing degree $\mathbf{d}$, which does not have a computable isomorphic copy. Downey, Seetapun and Knight [18] extended this result to an arbitrary nonzero Turing degree. Downey asked if there are familiar mathematical structures, such as linear orders, whose Turing degree spectra consist exactly of all nonzero Turing degrees. This question remains open. However, Miller has a partial answer. 
Theorem 4.1 (Miller [64]). There is a linear order whose Turing degree spectrum does not include $\mathbf{0}$, but includes all nonzero $\Delta_{2}$ Turing degrees.

Miller's proof uses the technique of permitting below a $\Delta_{2}$ set.

A countable structure $\mathcal{A}$ is automorphically trivial if there is a finite subset $F$ of the domain $A$ such that every permutation of $A$, whose restriction on $F$ is the identity, is an automorphism of $\mathcal{A}$. For example, every infinite linear order is automorphically nontrivial. Knight [47] proved that for an automorphically nontrivial structure $\mathcal{A}$, and a Turing degree $\mathbf{d}$ with $\mathbf{d} \geq$ $\operatorname{deg}(\mathcal{A})$, there is a structure $\mathcal{B} \cong \mathcal{A}$ such that $\operatorname{deg}(\mathcal{B})=\mathbf{d}$. That is, $\operatorname{Dg} \operatorname{Sp}(\mathcal{A})$ is closed upwards. On the other hand, for an automorphically trivial structure, all isomorphic copies have the same Turing degree. Building on Knight's result, we can show that for any (countable) structure, there is a copy in which the complete diagram, and all of the $n$-diagrams have the same Turing degree.

TheOREM 4.2 (Harizanov, Knight and Morozov [32]). For every automorphically trivial structure $\mathcal{A}$, we have $D^{c}(\mathcal{A}) \equiv_{T} D_{0}(\mathcal{A})$. For every automorphically nontrivial structure $\mathcal{A}$, and every set $X \geq_{T} D^{c}(\mathcal{A})$, there exists $\mathcal{B} \cong \mathcal{A}$ such that

$$
D^{c}(\mathcal{B}) \equiv_{T} D_{0}(\mathcal{B}) \equiv_{T} X .
$$

The following result shows that whenever a structure with a certain interesting Turing degree spectrum is found, then there are such structures within some well-known classes of algebraic structures.

Theorem 4.3 (Hirschfeldt, Khoussainov, Shore and Slinko [36]). For every automorphically nontrivial (countable) structure $\mathcal{G}$, there is a symmetric irreflexive graph, a partial order, a lattice, a ring, an integral domain of arbitrary characteristic, a commutative semigroup, and a 2-step nilpotent group, $\mathcal{A}$, such that $\operatorname{DgSp}(\mathcal{A})=\operatorname{DgSp}(\mathcal{G})$.

Since the Turing degree of a structure is not invariant under isomorphisms, Jockusch introduced the following complexity measure of the isomorphism type of a structure.

Definition 3. The (Turing) degree of the isomorphism type of $\mathcal{A}$, if it exists, is the least Turing degree in $\operatorname{Dg} \operatorname{Sp}(\mathcal{A})$.

The next theorem establishes that the Turing degree of the isomorphism type of a structure without a computable copy, which satisfies a certain condition on effective extendability of embeddings, does not exist. We say that a structure $\mathcal{A}$ satisfies the effective extendability condition if for every finite structure $\mathcal{C}$ isomorphic to a substructure of $\mathcal{A}$, and every embedding $f$ of $\mathcal{C}$ into $\mathcal{A}$, there is an algorithm that determines whether a given finite structure $\mathcal{D}$ extending $\mathcal{C}$ can be embedded into $\mathcal{A}$ by an embedding extending $f$. (For example, Slaman and Soare [77] showed that the c.e. Turing degrees, with 
the usual order relation of Turing degrees, satisfy the effective extendability condition.)

THEOREM 4.4 (Richter [74]). Assume that a structure $\mathcal{A}$ satisfies the effective extendability condition. If the degree of the isomorphism type of $\mathcal{A}$ exists, then it must be $\mathbf{0}$.

For example, a (countable) linear order satisfies the effective extendability condition. Thus, a linear order that is not isomorphic to a computable one, does not have a degree of its isomorphism type. Also, the isomorphism class of a tree without a computable copy does not have a degree of its isomorphism type.

THEOREM 4.5 (Richter [74]). Let $T$ be a theory in a finite language $L$ such that there is a computable sequence $\mathcal{A}_{0}, \mathcal{A}_{1}, \mathcal{A}_{2}, \ldots$ of finite structures for $L$, which are pairwise nonembeddable. Assume that for every set $X \subseteq \omega$, there is a (countable) model $\mathcal{A}_{X}$ of $T$ such that $\mathcal{A}_{X} \leq_{T} X$, and for every $i \in \omega$,

$$
\mathcal{A}_{i} \text { is embeddable in } \mathcal{A}_{X} \Leftrightarrow i \in X .
$$

Then for every Turing degree $\mathbf{d}$, there is a (countable) model of $T$ whose isomorphism type has degree $\mathbf{d}$.

For example, as a corollary, we obtain that for every Turing degree d, there is an Abelian group whose isomorphism type has degree d. Similarly, we can prove that there is such a lattice. On the other hand, Richter [74] showed that a modification of the previous theorem, obtained by replacing Turing reducibility in $\mathcal{A}_{X} \leq_{T} X$ by the enumeration reducibility, yields a very different conclusion-that there is a set $X$ such that the isomorphism type of $\mathcal{A}_{X}$ does not have a degree. As a corollary, we obtain that there is a countable Abelian group whose isomorphism type does not have a degree.

Since many countable structures do not have a degree of their isomorphism type, Jockusch introduced the following measure of complexity of structures, which is invariant under isomorphisms.

Definition 4. Let $\alpha$ be a computable ordinal. The $\alpha$ th jump degree of a structure $\mathcal{A}$, if it exists, is the least Turing degree in $\left\{\operatorname{deg}(\mathcal{B})^{(\alpha)}: \mathcal{B} \cong \mathcal{A}\right\}$.

Obviously, the notion of the 0 th jump degree of $\mathcal{A}$ coincides with the notion of the degree of the isomorphism type of $\mathcal{A}$. No nonstandard model of $P A$ has 0 th jump degree. On the other hand, there is a nonstandard model of $P A$ with any first jump degree $\mathbf{d}^{\prime}$. Knight [47] showed that the only possible first jump degree for a linear order is $\mathbf{0}^{\prime}$. Downey and Knight, building on the previous work of Ash, Jockusch and Knight [4], established the following sharp result for the jump degrees of a linear order.

THEOREM 4.6 (Downey and Knight [17]). Let a Turing degree $\mathbf{d}$ be such that $\mathbf{d} \geq \mathbf{0}^{(\alpha)}$, where $\alpha \geq 1$ is a computable ordinal. There is a linear order $\mathcal{A}$ whose $\alpha$ th jump degree is $\mathbf{d}$, and such that $\mathcal{A}$ does not have $\beta$ th jump degree for any $\beta<\alpha$. 
Jockusch and Soare have established a sharp result for the jump degrees of a Boolean algebra.

TheOREm 4.7 (Jockusch and Soare [37]). Let $\mathbf{d}$ be a Turing degree, and $n \in \omega$.

(a) If a Boolean algebra has $n$th jump degree $\mathbf{d}$, then $\mathbf{d}=\mathbf{0}^{(n)}$.

(b) If $\mathbf{d} \geq \mathbf{0}^{(\omega)}$, then there is a Boolean algebra with $\omega$ th jump degree $\mathbf{d}$.

Csima's results on the complexity of prime models of a complete decidable theory resolve the jump degree question for such prime models.

THEOREM 4.8 (Csima [14], [13]). Let $\mathcal{A}$ be a prime model of a complete decidable theory with no computable prime model.

(a) The structure $\mathcal{A}$ does not have a 0 th jump degree.

(b) For every $n \in \omega-\{0\}$, $\mathcal{A}$ has the nth jump degree $\mathbf{0}^{(n)}$.

$\S 5$. -diagrams of countable structures. Ash and Nerode [7] defined an additional relation $R$ on the domain $A$ of a computable structure $\mathcal{A}$ to be intrinsically c.e. on $\mathcal{A}$ if on all computable copies $\mathcal{B}$ of $\mathcal{A}$, the image of $R$ is c.e. They showed that, under a suitable extra decidability condition, a relation is intrinsically c.e. on $\mathcal{A}$ if and only if it is formally c.e. on $\mathcal{A}$. A relation is formally c.e. on $\mathcal{A}$ if it is definable in $\mathcal{A}$ by a c.e. disjunction of existential formulae with finitely many fixed parameters. The Ash-Nerode decidability condition is expressed in terms of $\mathcal{A}$ and $R$, and follows from the decidability of the existential diagram of $(\mathcal{A}, R)$.

A relation $R$ is relatively intrinsically c.e. on $\mathcal{A}$ if for all copies $\mathcal{B}$ of $\mathcal{A}$, the image of $R$ is c.e. relative to $D_{0}(\mathcal{B})$. Not every intrinsically c.e. relation is relatively intrinsically c.e. The following relativized Ash-Nerode theorem does not require an additional decidability condition.

TheOREM 5.1 (Chisholm [11], Ash, Knight, Manasse and Slaman [3]). $A$ relation $R$ is formally c.e. on $\mathcal{A}$ iff $R$ is relatively intrinsically c.e. on $\mathcal{A}$.

While the proof of Ash-Nerode theorem uses the priority argument, the proof of the relativized Ash-Nerode theorem uses forcing. Both theorems can be extended within arithmetical and hyperarithmetical hierarchies (see [6]).

There are familiar structures $\mathcal{A}$ such that for all $\mathcal{B} \cong \mathcal{A}$, we have $D^{c}(\mathcal{B}) \equiv_{T}$ $D_{0}(\mathcal{B})$. In particular, this is true for algebraically closed fields, and for other structures for which we have effective elimination of quantifiers. In the following theorem, we give syntactic conditions on $\mathcal{A}$ under which for all $\mathcal{B} \cong \mathcal{A}$, we have $D^{c}(\mathcal{B}) \equiv_{T} D_{n}(\mathcal{B})$. This result can be obtained using forcing, as for the relativized Ash-Nerode theorem.

Theorem 5.2 (Harizanov, Knight and Morozov [32]). For a (countable) structure $\mathcal{A}$ and $n \in \omega$, the following are equivalent:

(i) For every structure $\mathcal{B} \cong \mathcal{A}$ we have

$$
D^{c}(\mathcal{B}) \leq_{T} D_{n}(\mathcal{B})
$$


(ii) There exist a finite sequence $\bar{c} \in A^{<\omega}$ and a computable function $d$ assigning to every formula $\gamma(\bar{x})$ a c.e. disjunction $d_{\gamma}(\bar{c}, \bar{x})$ of $\Sigma_{n+1}$ formulae with parameters $\bar{c}$, such that

$$
\mathcal{A}_{A}=(\forall \bar{x})\left[\gamma(\bar{x}) \Leftrightarrow d_{\gamma}(\bar{c}, \bar{x})\right] .
$$

For example, it will follow that if $\mathcal{B}$ is a linear order of type $\omega^{k} \cdot \eta$, where $\eta$ is the order type of rationals, then

$$
D^{c}(\mathcal{B}) \equiv_{T} D_{2 k}(\mathcal{B}) .
$$

Let $\mathcal{A}$ be a decidable linear order of type $\omega^{k} \cdot \eta$. For any formula $\gamma(\bar{x})$, we obtain $d_{\gamma}$ as follows. For every tuple $\bar{a}$, we can find a $\Sigma_{2 k+1}$ formula $\psi_{\bar{a}}(\bar{x})$ defining the orbit of $\bar{a}$ under the automorphisms of $\mathcal{A}$. Then $d_{\gamma}(\bar{x})$ is the disjunction of the formulae $\psi_{\bar{a}}(\bar{x})$, for $\bar{a}$ satisfying $\gamma(\bar{x})$ in $\mathcal{A}$.

We also have a general condition, analogous to the one in Theorem 5.2 (ii), for the collapse of the $(n+1)$-diagram to the $n$-diagram, $D_{n+1}(\mathcal{B}) \leq_{T} D_{n}(\mathcal{B})$. We just have to replace in (ii) "formula $\gamma(\bar{x})$ " by " $\Pi_{n+1}$ formula $\gamma(\bar{x})$ ".

Clearly, a countable structure is intrinsically relatively 1-decidable if and only if its $\Pi_{1}$-definable relations are formally c.e., uniformly in the defining formulae. Let $\mathcal{A}$ be a computable structure for $L$, and let $R$ be an additional computable relation on its domain. The following result gives a general sufficient condition for realizing every c.e. Turing degree as the image of $R$ in a computable isomorphic copy of $\mathcal{A}$.

Let $\bar{c}$ be a finite sequence of elements in $A$. We say that an element $a \in A-R$ is free over $\bar{c}$ if for every existential formula $\sigma(\bar{x}, u)$ with $\operatorname{lh}(\bar{x})=$ $\operatorname{lh}(\bar{c})$, in $L \cup\{R\}$ with only positive occurrences of $R$, if $\left(\mathcal{A}_{A}, R\right) \quad \sigma(\bar{c}, a)$, then there is $a^{\prime} \in R$ for which $\left(\mathcal{A}_{A}, R\right) \quad \sigma\left(\bar{c}, a^{\prime}\right)$.

THEOREM 5.3 (Harizanov [30]). Let $R$ be a computable relation on a computable structure $\mathcal{A}$. Assume that for every $\bar{c} \in A^{<\omega}$, we can effectively find $a \in A-R$ such that a is free over $\bar{c}$. Then for every c.e. set $Y \subseteq \omega$, there is an isomorphism $f$ from $\mathcal{A}$ onto a computable structure $\mathcal{B}$ such that $f(R)$ is c.e. and $f(R) \equiv_{T} Y$.

In [66], Moses investigated 1-diagrams of linear orders, and proved that a computable linear order is 1-decidable if and only if its successor relation is computable. Using quantifier elimination, Moses showed that in a linear order with a computable universe, the 1-diagram is computable relative to the order relation together with the successor relation. For example, it then follows from Theorem 5.3 that for every c.e. set $Y \subseteq \omega$, there is a computable linear order $\mathcal{B}$ of order type $\omega$ such that $D_{1}(\mathcal{B}) \equiv_{T} Y$.

The next result, which follows from the proofs in [32], gives a general sufficient condition for realizing arbitrary possible Turing degrees for the existential and atomic diagrams of isomorphic copies of a structure.

THEOREM 5.4. Let $\mathcal{A}$ be a (countable) structure satisfying the following conditions for every sequence $\bar{c} \in A^{<\omega}$. 
$\left(C_{1}\right)$ We can effectively find a $\Pi_{1}$ formula $\theta(\bar{x}, \bar{u})$ with $\operatorname{lh}(\bar{x})=\operatorname{lh}(\bar{c})$, and $a$ sequence $\bar{a} \in A^{\text {lh }(\bar{u})}$ such that $\mathcal{A}_{A}=\theta(\bar{c}, \bar{a})$, and for every $\Sigma_{1}$ formula $\sigma(\bar{x}, \bar{u})$, we have

$$
\left[\mathcal{A}_{A}=\sigma(\bar{c}, \bar{a})\right] \Rightarrow\left(\exists \bar{a}^{\prime} \in A^{l h(\bar{u})}\right)\left[\mathcal{A}_{A}=\left(\sigma\left(\bar{c}, \bar{a}^{\prime}\right) \wedge \neg \theta\left(\bar{c}, \bar{a}^{\prime}\right)\right)\right] .
$$

$\left(C_{0}\right)$ We can effectively find a $\Delta_{1}$ formula $\chi(\bar{x}, \bar{u})$, given by a pair of $\Sigma_{1}, \Pi_{1}$ formulae, such that for two sequences of distinct elements, $\bar{a}, \bar{a}^{\prime} \in A^{\operatorname{lh}(\bar{u})}$, disjoint from $\bar{c}$, we have

$$
\mathcal{A}_{A} \models \chi(\bar{c}, \bar{a}) \wedge \neg \chi\left(\bar{c}, \bar{a}^{\prime}\right) .
$$

Let the sets $X, Y \subseteq \omega$ be such that $X \geq_{T} D_{0}(\mathcal{A})$ and $Y$ is c.e.a. in $X$. Then there is a structure $\mathcal{B} \cong \mathcal{A}$ such that

$$
D_{0}(\mathcal{B}) \equiv_{T} X \wedge D_{1}(\mathcal{B}) \equiv_{T} Y .
$$

Thus, there is a linear order $\mathcal{B}$ of order type $\omega$ with $D_{0}(\mathcal{B}) \equiv_{T} X$ and $D_{1}(\mathcal{B}) \equiv_{T} Y$. A corresponding $\Pi_{1}$ formula uses the successor relation, and a corresponding $\Delta_{1}$ formula uses the order relation. Also, if $\mathcal{A}$ is the interval Boolean algebra of $(\omega,<)$, then there exists $\mathcal{B} \cong \mathcal{A}$ with $D_{0}(\mathcal{B}) \equiv_{T}$ $X$ and $D_{1}(\mathcal{B}) \equiv_{T} Y$. A corresponding $\Pi_{1}$ formula uses the unary atom relation, and a corresponding $\Delta_{1}$ formula uses the binary relation of being disjoint. Similarly, if $\mathcal{A}$ is the Abelian group $Z_{p}^{\omega} \oplus Z_{p^{2}}^{\omega}$, where $p$ is a prime number, then there exists $\mathcal{B} \cong \mathcal{A}$ with $D_{0}(\mathcal{B}) \equiv_{T} X$ and $D_{1}(\mathcal{B}) \equiv_{T} Y$. A corresponding $\Pi_{1}$ formula uses the unary relation of not being divisible by $p$, and a corresponding $\Delta_{1}$ formula uses the binary relation of one element being a multiple by $p$ of the other element.

If a structure $\mathcal{A}$ is 1 -decidable, then the condition $\left(C_{1}\right)$ in Theorem 5.4 allows the existence of a computable copy $\mathcal{B}$ of $\mathcal{A}$ such that $D_{1}(\mathcal{B})$ is of an arbitrary c.e. degree. We next investigate Turing degrees of all $n$-diagrams of isomorphic copies of a structure.

TheOREM 5.5 (Chisholm and Moses [12]). There is a structure $\mathcal{A}$ that is $n$ decidable and whose every computable copy is $n$-decidable, for all $n$, but $\mathcal{A}$ has no decidable copy.

Knight extended Theorem 3.3 by characterizing possible sequences of Turing degrees of $n$-diagrams of nonstandard models of $P A$.

TheOREM 5.6 (Knight [46]). Let $\left(\mathbf{d}_{n}\right)_{n \in \omega}$ be an infinite sequence of Turing degrees. Then the following are equivalent:

(i) There exists a nonstandard model $\mathcal{A}$ of PA such that

$$
(\forall n)\left[\operatorname{deg}\left(D_{n}(\mathcal{A})=\mathbf{d}_{n}\right]\right.
$$

(ii) There exists an $\omega$-table $\left(C_{n}\right)_{n \in \omega}$ over a completion of $P A$, such that

$$
(\forall n)\left[\operatorname{deg}\left(C_{n}\right)=\mathbf{d}_{n}\right] .
$$


We do not know what possible sequences of Turing degrees are for $\left(D_{n}(\mathcal{B})\right)_{n \in \omega}$, where $\mathcal{B} \cong \mathcal{N}$.

Let $\mathcal{A}$ be a structure. For a single tuple $\bar{a}$ or concatenated tuples $\bar{a}, \bar{c}$ from $A$, we assume that their elements are all distinct. The standard backand-forth relations $\leq_{\alpha}$ can be defined for arbitrary countable ordinals $\alpha$. However, we give a definition in the case when $\alpha$ is finite, and, for simplicity, consider only structures $\mathcal{A}$ for a finite relational language. This assumption simplifies the definition of $\leq_{0}$.

Definition 5 (Barwise [8], Ash and Knight [6]). Let $\bar{a}, \bar{b}$ be tuples from $A^{<\omega}$ such that $\operatorname{lh}(\bar{a}) \leq \operatorname{lh}(\bar{b})$. Then

(i) $\bar{a} \leq_{0} \bar{b}$ if the open formulae true of $\bar{a}$ are true of $\bar{b}$;

(ii) $\bar{a} \leq_{n+1} \bar{b}$ if for every $\bar{d}$, there exists $\bar{c}$ such that $\bar{b}, \bar{d} \leq_{n} \bar{a}, \bar{c}$.

The following notion of independence from [32] uses the back-and-forth relations and plays a fundamental role in the theorem that follows.

DeFINITION 6. Let $\bar{u}$ be a tuple of distinct variables.

(i) The formula $\theta(\bar{u}, \bar{x})$ is 0 -independent over $\bar{u}$ if it is open, and for every $\bar{c} \in A^{l h(\bar{u})}$, there exist $\bar{a}, \bar{a}^{\prime} \in A^{\operatorname{lh}(\bar{x})}$ such that

$$
\mathcal{A}_{A} \models \theta(\bar{c}, \bar{a}) \text { and } \mathcal{A}_{A} \models \neg \theta\left(\bar{c}, \bar{a}^{\prime}\right) .
$$

(ii) For $n>0$, the formula $\theta(\bar{u}, \bar{x})$ is $n$-independent over $\bar{u}$ if it is $\Pi_{n}$ and for every $\bar{c} \in A^{\operatorname{lh}(\bar{u})}$,

- there exists $\bar{a}$ such that $\mathcal{A}_{A}=\theta(\bar{c}, \bar{a})$, and

- for every $\bar{a}$ with $\mathcal{A} \models \theta(\bar{c}, \bar{a})$, and any $\bar{a}_{1}$, there exist $\bar{a}^{\prime}$ and $\bar{a}_{1}^{\prime}$ such that

$$
\mathcal{A}=\neg \theta\left(\bar{c}, \bar{a}^{\prime}\right) \text { and } \bar{c}, \bar{a}, \bar{a}_{1} \leq_{n-1} \bar{c}, \bar{a}^{\prime}, \bar{a}_{1}^{\prime} .
$$

In linear orders of order type $\omega^{\omega}$ or $\omega^{\omega} \cdot \eta$, we use the $n$-independent formulae similar to the ones used by Moses in [67]. The $n$-independent formula over $\bar{u}$ says that $x$ is greater than any element in $\bar{u}$; and $x<y$ if $n=0, y$ is the successor of $x$ if $n=1, x$ is a 1-limit if $n=2, x$ and $y$ are 1-limits and $y$ is the next one after $x$ if $n=3$, etc.

It will follow from the following general theorem that the sequences of Turing degrees of $n$-diagrams of linear orders of the order type $\omega^{\omega}$ or $\omega^{\omega} \cdot \eta$ coincide with the sequences of Turing degrees of $\omega$-tables. The theorem is proved using Ash's method of $\alpha$-systems, a framework for nested priority constructions developed by Ash and Knight (see [6]). Our proof exploits a metatheorem from [46], for coding and enumerating $\alpha$-systems for $\alpha \leq \omega$. The metatheorem provides a list of "abstract" conditions guaranteeing the success of a priority construction.

THEOREM 5.7 (Harizanov, Knight and Morozov [32]). Using the notions above, assume that $\mathcal{A}$ is decidable, and that the relations $\leq_{n}$ are c.e. for $n \in \omega$, uniformly in $n$. Assume also that for every $n$ and every tuple $\bar{u}$ of variables, we can effectively find a formula that is $n$-independent over $\bar{u}$. Then for any 
$\omega$-table $\left(C_{n}\right)_{n<\omega}$, there exists $\mathcal{B} \cong \mathcal{A}$ such that

$$
D_{n}(\mathcal{B}) \equiv_{T} C_{n}
$$

for $n \in \omega$, uniformly in $n$.

The uniformity implies that $D^{c}(\mathcal{B}) \equiv \oplus_{n<\omega} C_{n}$.

A theorem analogous to the previous one can be established for any $(k+1)$ table $\left(C_{n}\right)_{n<k}$, where $1 \leq k<\omega$. It is enough to assume that $\mathcal{A}$ is $k$ decidable, that the relations $\leq_{0}, \ldots, \leq_{k-1}$ are c.e., and that for every $\bar{u}$ and every $n \leq k$, we can effectively find a formula that is $n$-independent over $\bar{u}$. For example, for any $(2 m)$-table $\left(C_{n}\right)_{n \leq 2 m-1}$, where $1 \leq m<\omega$, there is a linear order of the order type $\omega^{m}$ such that $D_{n}(\mathcal{B}) \equiv_{T} C_{n}$ for every $n \leq 2 m-1$.

In addition to linear orders, it would be worthwhile to investigate possible sequences of Turing degrees of $n$-diagrams for other natural mathematical structures. For models of completions of $P A$, see [32] for a conjecture about a characterization of these sequences.

\section{REFERENCES}

[1] A. Arana, Possible m-diagrams of models of arithmetic, to appear in a volume on reverse mathematics (S. G. Simpson, editor).

[2] - Solovay's theorem cannot be simplified, Annals of Pure and Applied Logic, vol. 112 (2001), pp. 27-41.

[3] C. Ash, J. Knight, M. Manasse, and T. Slaman, Generic copies of countable structures, Annals of Pure and Applied Logic, vol. 42 (1989), pp. 195-205.

[4] C. J. Ash, C. G. Jockusch, JR., and J. F. KNIGHT, Jumps of orderings, Transactions of the American Mathematical Society, vol. 319 (1990), pp. 573-599.

[5] C. J. Ash and J. F. Knight, Possible degrees in recursive copies II, Annals of Pure and Applied Logic, vol. 87 (1997), pp. 151-165.

[6] Computable structures and the hyperarithmetical hierarchy, Elsevier, Amsterdam, 2000.

[7] C. J. AsH and A. Nerode, Intrinsically recursive relations, Aspects of effective algebra (J. N. Crossley, editor), Upside Down A Book Co., Yarra Glen, Australia, 1981, pp. 26-41.

[8] J. BARWISE, Back and forth through infinitary logic, Studies in model theory (M. D. Morley, editor), MAA Studies in Mathematics 8, Buffalo, New York, 1973, pp. 5-34.

[9] S. BuECHLER, Essential stability theory, Springer-Verlag, Berlin, 1996.

[10] C. C. Chang and H. J. KeIsLer, Model theory, North-Holland, Amsterdam, 1990.

[11] J. Chisholm, Effective model theory vs. recursive model theory, The Journal of Symbolic Logic, vol. 55 (1990), pp. 1168-1191.

[12] J. Chisholm and M. Moses, An undecidable linear order that is $n$-decidable for all $n$, Notre Dame Journal of Formal Logic, vol. 39 (1998), pp. 519-526.

[13] B. F. Csima, Computability of prime and saturated models, Ph.D. thesis, University of Chicago, in preparation.

[14] - Degree spectra of prime models, Abstracts of papers presented to the American Mathematical Society, vol. 23 (1), 2002, (Joint Mathematics Meetings, San Diego, January 6-9, 2002), p. 14.

[15] B. F. Csima, D. R. Hirschfeldt, J. F. Knight, and R. I. SoAre, Bounding prime models, preprint. 
[16] R. Downey and C. G. JockUSCH, JR., Every low Boolean algebra is isomorphic to a recursive one, Proceedings of the American Mathematical Society, vol. 122 (1994), pp. 871880.

[17] R. Downey and J. F. KNIGHT, Orderings with $\alpha$ th jump degree $\boldsymbol{0}^{(\alpha)}$, Proceedings of the American Mathematical Society, vol. 114 (1992), pp. 545-552.

[18] R. G. Downey, On presentations of algebraic structures, Complexity, logic, and recursion theory (A. Sorbi, editor), Lecture Notes in Pure and Applied Mathematics 187, Marcel Dekker, New York, 1997, pp. 157-205.

[19] YU. L. Ershov, Decidability problems and constructive models, Nauka, Moscow, 1980, Russian.

[20] Yu. L. Ershov and S. S. Goncharov, Constructive models, Siberian School of Algebra and Logic, Kluwer Academic/Plenum Publishers, 2000, English translation.

[21] S. Feferman, Arithmetically definable models of formalized arithmetic, Notices of the American Mathematical Society, vol. 5, 1958, pp. 679-680.

[22] S. S. Goncharov, Restricted theories of constructive Boolean algebras, Sibirskii Matematicheskii Zhurnal, vol. 17 (1976), pp. 601-611, English translation.

[23] - Constructive models of $\aleph_{1}$-categorical theories, Mathematical Notes, vol. 23 (1978), pp. 885-888, Russian.

[24] - Strong constructivizability of homogeneous models, Algebra and Logic, vol. 17 (1978), pp. 247-263, English translation.

[25] - A totally transcendental decidable theory without constructivizable homogeneous models, Algebra and Logic, vol. 19 (1980), pp. 85-93, English translation.

[26] - Countable Boolean algebras and decidability, Siberian School of Algebra and Logic, Consultants Bureau, New York, 1997.

[27] S. S. Goncharov, V. S. Harizanov, M. C. Laskowski, S. LempP, and C. F. D. McCoy, Trivial, strongly minimal theories are model complete after naming constants, Proceedings of the American Mathematical Society, (to appear).

[28] S. S. Goncharov and B. M. Khoussainov, Complexity of theories of computable models, Algebra and Logic, (to appear).

[29] S. S. Goncharov and A. T. Nurtazin, Constructive models of complete solvable theories, Algebra and Logic, vol. 12 (1973), pp. 67-77, English translation.

[30] V. S. Harizanov, Some effects of Ash-Nerode and other decidability conditions on degree spectra, Annals of Pure and Applied Logic, vol. 55 (1991), pp. 51-65.

[31] - Pure computable model theory, Handbook of recursive mathematics (Yu. L. Ershov, S. S. Goncharov, A. Nerode, J. B. Remmel, and V. W. Marek (assoc.), editors), vol. 1, Elsevier, Amsterdam, 1998, pp. 3-114.

[32] V. S. Harizanov, J. F. Knight, and A. S. Morozov, Sequences of n-diagrams, The Journal of Symbolic Logic, (to appear).

[33] L. HARRINGTON, Recursively presentable prime models, The Journal of Symbolic Logic, vol. 39 (1974), pp. 305-309.

[34] B. Herwig, S. LEMPP, and M. ZIEGLER, Constructive models of uncountably categorical theories, Proceedings of the American Mathematical Society, vol. 127 (1999), pp. 3711-3719.

[35] D. R. HiRsChFELDT, Prime models of theories of computable linear orderings, Proceedings of the American Mathematical Society, vol. 129 (2001), pp. 3079-3083.

[36] D. R. Hirschfeldt, B. Khoussainov, R. A. Shore, and A. M. Slinko, Degree spectra and computable dimension in algebraic structures, Annals of Pure and Applied Logic, vol. 115 (2002), pp. 71-113.

[37] C. G. Jockusch and R. I. Sonre, Boolean algebras, Stone spaces, and the iterated Turing jump, The Journal of Symbolic Logic, vol. 59 (1994), pp. 1121-1138.

[38] C. G. JockUSCH JR. and R. I. SOARE, $\Pi_{1}^{0}$ classes and degrees of theories, Transactions of the American Mathematical Society, vol. 173 (1972), pp. 33-56. 
[39] - Degrees of orderings not isomorphic to recursive linear orderings, Annals of Pure and Applied Logic, vol. 52 (1991), pp. 39-64.

[40] R. KaYe, Models of Peano Arithmetic, Clarendon Press, Oxford, 1991.

[41] N. G. Khisamiev, On strongly constructive models of a decidable theory, Izvestii Akademii Nauk Kazakhstan SSR, vol. 35, Ser. Fiz.-Mat., no. 1, 1974, Russian, pp. 83-84.

[42] B. Khoussainov, A. NiEs, and R. A. SHore, Computable models of theories with few models, Notre Dame Journal of Formal Logic, vol. 38 (1997), pp. 165-178.

[43] B. Khoussainov and R. A. SHORE, Effective model theory: the number of models and their complexity, Models and computability, invited papers from Logic Colloquium '97 (S. B. Cooper and J. K. Truss, editors), London Mathematical Society Lecture Notes Series 259, Cambridge University Press, Cambridge, 1999, pp. 193-239.

[44] J. Knight, A. H. Lachlan, and R. I. Soare, Two theorems on degrees of models of true arithmetic, The Journal of Symbolic Logic, vol. 49 (1984), pp. 425-436.

[45] J. F. KNIGHT, Models of arithmetic: quantifiers and complexity, (S. G. Simpson, editor), to appear in a volume on reverse mathematics.

[46] - Sequences of degrees associated with models of arithmetic, to appear in a volume on Logic Colloquium 2001.

[47] — Degrees coded in jumps of orderings, The Journal of Symbolic Logic, vol. 51 (1986), pp. 1034-1042.

[48] - True approximations and models of arithmetic, Models and computability, invited papers from Logic Colloquium '97 (S. B. Cooper and J. K. Truss, editors), London Mathematical Society Lecture Note Series 259, Cambridge University Press, 1999, pp. 255 278.

[49] - Minimality and completions of PA, The Journal of Symbolic Logic, vol. 66 (2001), pp. 1447-1457.

[50] J. F. Knight and M. Sтов, Computable Boolean algebras, The Journal of Symbolic Logic, vol. 65 (2000), pp. 1605-1623.

[51] K. ZH. KudaIBergenov, Constructivizable models of undecidable theories, Siberian Mathematical Journal, vol. 21 (1980), no. 5, pp. 155-158, Russian.

[52] A. H. LACHLAN and R. I. SOARE, Models of arithmetic and upper bounds for arithmetic sets, The Journal of Symbolic Logic, vol. 59 (1994), pp. 977-983.

[53] - Models of arithmetic and subuniform bounds for the arithmetic sets, The Journal of Symbolic Logic, vol. 63 (1998), pp. 59-72.

[54] M. LeRman and J. H. SCHMERL, Theories with recursive models, The Journal of Symbolic Logic, vol. 44 (1979), pp. 59-76.

[55] A. MacintYre and D. Marker, Degrees of recursively saturated models, Transactions of the American Mathematical Society, vol. 282 (1984), pp. 539-554.

[56] D. Marker, Degrees of models of true arithmetic, Proceedings of the Herbrand symposium, Logic Colloquium '81 (J. Stern, editor), North-Holland, Amsterdam, 1982, pp. 233 242.

[57] - Non $\Sigma_{n}$ axiomatizable almost strongly minimal theories, The Journal of Symbolic Logic, vol. 54 (1989), pp. 921-927.

[58] G. Metakides and A. Nerode, Recursively enumerable vector spaces, Annals of Mathematical Logic, vol. 11 (1977), pp. 147-171.

[59] — Effective content of field theory, Annals of Mathematical Logic, vol. 17 (1979), pp. $289-320$.

[60] T. Millar, Homogeneous models and decidability, Pacific Journal of Mathematics, vol. 91 (1980), pp. 407-418.

[61] - Omitting types, type spectrums, and decidability, The Journal of Symbolic Logic, vol. 48 (1983), pp. 171-181. 
[62] T. S. Millar, Foundations of recursive model theory, Annals of Mathematical Logic, vol. 13 (1978), pp. 45-72.

[63] - Pure recursive model theory, Handbook of computability theory (E. R. Griffor, editor), Elsevier, Amsterdam, 1999, pp. 507-532.

[64] R. MilleR, The $\Delta_{2}^{0}$-spectrum of a linear order, The Journal of Symbolic Logic, vol. 66 (2001), pp. 470-486.

[65] M. MORLEY, Decidable models, Israel Journal of Mathematics, vol. 25 (1976), pp. 233240.

[66] M. Moses, Recursive linear orders with recursive successivities, Annals of Pure and Applied Logic, vol. 27 (1984), pp. 253-264.

[67] - n-recursive linear orders without $(n+1)$-recursive copies, Logical methods (J. N. Crossley, J. B. Remmel, R. A. Shore, and M. E. Sweedler, editors), Birkhäuser, Boston, 1993, pp. 572-592.

[68] A. NIES, A new spectrum of recursive models, Notre Dame Journal of Formal Logic, vol. 40 (1999), pp. 307-314.

[69] M. Peretyat'KIn, Turing machine computations in finitely axiomatizable theories, Algebra and Logic, vol. 21 (1982), pp. 272-295, English translation.

[70] M. G. Peretyat'Kin, Criterion for strong constructivizability of a homogeneous model, Algebra and Logic, vol. 17 (1978), pp. 290-301, English translation.

[71] J. B. Remmel, Maximal and cohesive vector spaces, The Journal of Symbolic Logic, vol. 42 (1977), pp. 400-418.

[72] - Recursively enumerable Boolean algebras, Annals of Mathematical Logic, vol. 15 (1978), pp. 75-107.

[73] — Recursive isomorphism types of recursive Boolean algebras, The Journal of Symbolic Logic, vol. 46 (1981), pp. 572-594.

[74] L. J. Richter, Degrees of structures, The Journal of Symbolic Logic, vol. 46 (1981), pp. $723-731$.

[75] D. ScotT, Algebras of sets binumerable in complete extensions of arithmetic, Proceedings of the fifth symposium in pure mathematics (J. C. E. Dekker, editor), Recursive Function Theory, American Mathematical Society, Providence, Rhode Island, 1962, pp. 117-121.

[76] T. A. Slaman, Relative to any nonrecursive set, Proceedings of the American Mathematical Society, vol. 126 (1998), pp. 2117-2122.

[77] T. A. Slaman and R. I. SOARe, Extension of embeddings in the computably enumerable degrees, Annals of Mathematics, vol. 154 (2001), pp. 1-43.

[78] R. I. SOARE, Recursively enumerable sets and degrees, Springer-Verlag, Berlin, 1987.

[79] J. J. Thurber, Every low 2 Boolean algebra has a recursive copy, Proceedings of the American Mathematical Society, vol. 123 (1995), pp. 3859-3866.

[80] S. WeHner, Enumerations, countable structures, and Turing degrees, Proceedings of the American Mathematical Society, vol. 126 (1998), pp. 2131-2139.

\section{DEPARTMENT OF MATHEMATICS \\ THE GEORGE WASHINGTON UNIVERSITY \\ WASHINGTON, D.C. 20052, U.S.A.}

E-mail: harizanv@gwu.edu 